\section{Vigilância Sanitária: uma proposta de análise dos contextos locais}

\section{Sanitary Surveillance: a proposal for analyzing local environments}

\section{Márcia Franke Piovesan ${ }^{1 *}$ \\ Maria Valéria Vasconcelos Padrão² \\ Maria Umbelina Dumont ${ }^{2}$ \\ Gracia Maria Gondim ${ }^{3}$ \\ Oviromar Flores 4 \\ José Ivo Pedrosa ${ }^{5}$ \\ Luiz Felipe Moreira Lima ${ }^{6}$}

'Diretoria de Desenvolvimento Setorial, Agência Nacional de Saúde Suplementar

${ }^{2}$ Assessoria de Descentralização (Adavs), Agência Nacional de Vigilância Sanitária

${ }^{3}$ Núcleo de Estudos em Saúde Coletiva NESC, Universidade Federal do Rio Grande do Norte

${ }^{4}$ Departamento de Estudos em Saúde Coletiva, Faculdade de Ciências da Saúde, Universidade de Brasília

${ }^{5}$ Coordenação Geral de Ações Populares de Educação na Saúde, Secretaria do Trabalho e da Educação na Saúde, Ministério da Saúde

${ }^{6}$ Coordenação de Portos, Aeroportos e Fronteiras no Rio de Janeiro, Agência Nacional de Vigilância Sanitária

*Correspondência: Rua Augusto Severo, 83 Glória, 20021-040 - Rio de Janeiro, RJ, marcia.piovesan@ans.gov.br

\section{Resumo}

O trabalho apresenta pesquisa realizada em oito municípios do Estado da Paraíba, com o objetivo de analisar a relação entre os serviços de Vigilância Sanitária e os contextos sanitário, epidemiológico, político, social e econômico de seus territórios. O estudo, concluído em julho de 2003, foi desenvolvido pela Assessoria de Descentralização da Agência Nacional de Vigilância Sanitária (ANVISA) e integra o Projeto Redevisa (Rede Descentralizada de Vigilância Sanitária), cuja proposta incluía a identificação de prioridades sanitárias e epidemiológicas locais e regionais para o repasse de recursos financeiros pela ANVISA. A pesquisa envolveu a visita aos municípios para a coleta de dados e informações, e seus resultados foram analisados à luz de forças restritivas e impulsoras para o desempenho dos Serviços de Vigilância Sanitária sob os aspectos relacionados à estrutura, processos de trabalho, gestão, contexto político e recursos financeiros. $\mathrm{O}$ estudo constatou a deficiente articulação entre o trabalho das Vigilâncias pesquisadas e o espaço sobre o qual atuam, tendo identificado fatores restritivos importantes para a ação de controle sanitário local. Neste artigo são expostas as idéias norteadoras da pesquisa, os resultados da análise, bem como o método proposto de reconhecimento e sistematização das informações indispensáveis para o planejamento em Vigilância Sanitária.

Palavras-chave: Vigilância Sanitária. Planejamento em saúde. Epidemiologia. 


\section{Abstract}

This paper presents the results of a study carried out in eight municipalities of the state of Paraíba with the objective of analyzing the relationship between local services and the sanitary, epidemiological, political, social and economic contexts of these territories. The study, completed in July 2003, was developed by the Advisory Office for Decentralization of Sanitary Surveillance Actions from the National Health Surveillance Agency (ANVISA), and integrates the Redevisa Project (Sanitary Surveillance Decentralized Networking), whose proposal involves the identification of local and regional sanitary and epidemiological priorities for ANVISA to pass on financial resources. The research involved visits to municipalities to collect data and information. Results were analyzed in the light of both restricting and facilitating forces related to the delivery of Sanitary Surveillance Services, in terms of structure, work processes, management, political context and financial resources. The study explicitly revealed the deficient interaction between the work of the Sanitary Surveillance authorities researched and the environment where they operate, and identified important restrictive factors for local sanitary control. This article presents the driving ideas of the research, the results from the analysis, as well as the proposed approach for the recognition and systematization of indispensable information for Sanitary Surveillance planning.

Key Words: Sanitary Surveillance. Health planning. Epidemiology.

\section{Introdução}

As mudanças na política social do país, especialmente na área da saúde, a partir de 1990, introduziram desafios quanto à forma de planejar, gerir e avaliar as políticas de saúde em contextos descentralizados e autônomos, os quais exigem articulação intersetorial e intergovernamental e o incremento da participação da comunidade nas decisões do setor.

No campo da Vigilância Sanitária (VISA), a descentralização está estreitamente ligada à articulação entre as instâncias do Sistema Nacional de Vigilância Sanitária, que, apesar de autônomas, são interdependentes, e por isso, necessariamente cooperativas. Autonomia e interdependência na construção de um sistema descentralizado imprimem novas estratégias ao planejamento, à gestão e à avaliação das políticas de Vigilância Sanitária.

Entre os fatores que reduzem a efetividade das ações de controle sanitário são citados, com freqüência: atribuições pouco definidas das instâncias de governo; abordagem fragmentada do campo de atuação; pouca articulação intra e interinstitucional; insuficiência de recursos humanos; baixa qualificação técnica dos profissionais; sistema de informações insuficiente, além de despreparo para utilização dos dados existentes; interferência político-partidária; falta de apoio político, assim como desmobilização e desinformação da sociedade ${ }^{1}$.

Além dessas dificuldades, enfrentadas pelas Vigilâncias Sanitárias municipais, este estudo identificou o desconhecimento das prioridades dos seus próprios territórios ${ }^{2}$, o que potencializa a desvinculação entre prioridades locais e metas pactuadas, ignorando que, no campo da Vigilância Sanitária, os fatores de risco, as relações sociais e as políticas desenham os territórios e, em conseqüência disso, a efetividade dos serviços.

A aproximação entre território, fatores de risco e planejamento, aqui proposta, mediada pela presença e participação de atores sociais, constitui uma alternativa de planejamento que parte de problemas concretos, 
em territórios determinados e numa perspectiva intersetorial ${ }^{3}$.

A identificação de fatores de risco, compreendidos como as circunstâncias do ambiente ou características das pessoas que conferem maior ou menor probabilidade de acometimento de dano à saúde ${ }^{4}$, é fundamental no reconhecimento da Vigilância Sanitária como um conjunto de ações de Saúde Pública, voltadas para a promoção e proteção da saúde, mediante o controle sanitário dos processos, produtos e serviços de interesse da saúde, do meio ambiente e dos ambientes de trabalho ${ }^{5,6}$.

No trabalho de controle sanitário é fundamental conhecer o território, ou seja, identificar e interpretar a organização e a dinâmica das populações que nele habitam, bem como compreender a forma como funcionam e se articulam as condições econômicas, sociais e culturais, quais os atores sociais em questão e a relação destes com seus espaços de vida e de trabalho ${ }^{3,7}$. A identificação destes fatores possibilita minimizar os problemas sanitários a que estão expostos indivíduos, grupos sociais e objetos, e agir sobre os fatores que determinam e condicionam a ocorrência de agravos e danos ${ }^{8}$.

A análise das informações produzidas sobre um território subsidia o planejamento, que constitui importante instrumento de definição de prioridades, direcionamento de estratégias e efetivação das ações necessárias para a integralidade e eqüidade das políticas sociais, inclusive as de Vigilância Sanitária $^{9,10}$.

\section{Objetivos}

Algumas perguntas foram o ponto de partida para a pesquisa: a) as avaliações de estrutura e processos de trabalho dos Serviços de Vigilância Sanitária são suficientes para explicar seus resultados? b) a atual estratégia de descentralização leva em conta a realidade sanitária e epidemiológica dos municípios, bem como o conjunto de atores sociais que compõe os territórios nos quais a Vigilância atua? c) os recursos destinados aos Estados estão direcionados para as prio- ridades de controle sanitário dos municípios? d) as prioridades são conhecidas e sistematizadas pela Agência Nacional de Vigilância Sanitária (ANVISA), pelos Estados e municípios na definição das metas para o repasse de recursos?

Ao tempo em que estes questionamentos traduziam preocupações quanto ao direcionamento de recursos para prioridades locais, o Ministério Público de um município do Estado da Paraíba encaminhou denúncia a ANVISA, sobre a não aplicação dos recursos do Piso de Atenção Básica (PAB/VISA) nas ações de controle sanitário local. A denúncia desencadeou uma ação conjunta entre a ANVISA e a Agência Estadual de Vigilância Sanitária da Paraíba (AGEVISA/PB), e suscitou o planejamento de estratégias de descentralização no Estado, voltadas para as prioridades locais. A disposição da AGEVISA em analisar e enfrentar os fatores restritivos e impulsores envolvidos na ação dos Serviços de Vigilância Sanitária dos municípios foi decisivo para que o estudo se desenvolvesse na Paraíba ${ }^{2}$.

Dos 223 municípios, a equipe da AGEVISA/PB selecionou João Pessoa, Campina Grande, Cabedelo, Bayeux, Santa Rita, Soledade, Juazeirinho e Patos, definidos, pela Agência estadual, como representativos da complexidade produtiva e social e dos problemas epidemiológicos e ambientais do Estado, compreendendo cerca de $38,27 \%$ da sua população ${ }^{11}$.

Os contextos dos Serviços de Vigilância Sanitária dos oito municípios foram o objeto da pesquisa relatada neste artigo, executada por uma equipe de técnicos da ANVISA e da AGEVISA - que participou desde a definição do escopo até a articulação das agendas de compromisso com os municípios, para enfrentamento dos problemas identificados. A equipe foi posteriormente ampliada com consultores ligados à Universidade de Brasília (UnB), Universidade Federal do Piauí (UFPI) e Escola Politécnica Joaquim Venâncio (EPJV/ FIOCRUZ), para a elaboração e realização das oficinas de planejamento participativo em Vigilância Sanitária no Estado. 


\section{Metodologia}

O planejamento participativo tem como pressuposto que a situação de saúde compõe-se de um conjunto de aspectos referentes a objetivos (temas, problemas), contextos (atores, circunstâncias) e capacidades (recursos econômicos, políticos, técnicos e organizativos) em permanente mudança, em que o conhecimento para intervir sobre problemas e necessidades é constantemente construído.

Nessa construção, as operações que viabilizam as mudanças são distribuídas em três momentos: a) o momento explicativo: análise da situação de saúde; b) o momento estratégico; e c) o momento operativo, que podem ocorrer concomitantemente. Para subsidiar o momento explicativo do planejamento, e aproximá-lo da realidade local, propôs-se analisar a relação entre os Serviços de Vigilância Sanitária e os contextos sanitário, epidemiológico, político, social e econômico de seus territórios, por meio da Estimativa Rápida Participativa (ERP), que permite sistematizar dados em categorias bem definidas, específicas e facilmente gerenciáveis, além de expressar claramente as diferenças em termos de condições de vida e saúde dos diferentes grupos sociais ${ }^{12}$.

Trata-se de uma metodologia que não é recente, nem a única utilizada para informar o processo de planejamento participativo. Entretanto, este se beneficia da ERP, não somente por ser uma abordagem simples, rápida e de baixo custo, mas por envolver setores e organizações que participam do problema. Sua utilização apóia o planejamento participativo porque contribui para a identificação das condições de vida da população. Além disso, apresenta como resultado um conjunto de necessidades específicas, estruturadas a partir da própria população, em conjunto com técnicos e gestores de saúde, além de intervir tanto na esfera dos dirigentes e usuários da Vigilância Sanitária como na esfera das relações de poder entre atores sociais, estimulando a participação e o compromisso das partes envolvidas $^{13,14}$.
A Estimativa Rápida Participativa se apóia em três princípios: a) coleta de dados pertinentes e necessários; b) coleta de informações que reflitam condições locais e situações específicas; e c) envolvimento da comunidade na definição de seus próprios problemas e na busca de soluções, o que contribui para maior aceitação das intervenções posteriores. Trabalha fundamentalmente com três fontes de dados: a) registros existentes, de fontes primárias e secundárias; b) entrevistas com informantes-chave, freqüentemente usando pesquisas e questionários curtos; e c) observação de campo $^{12,13,14}$.

Com base neste referencial, o estudo realizado nos municípios da Paraíba buscou atender duas demandas fundamentais: a) necessidade de construir conhecimento estruturado dos principais problemas do campo da VISA, com o objetivo de fornecer diretrizes para o planejamento; e b) necessidade de construir este conhecimento de modo participativo, cuja prática do diagnóstico constituísse também um momento de reflexão sobre o território. Dessa maneira, o planejamento refletiria não somente a abordagem técnica, mas também o esforço coletivo dos atores sociais na resolução de seus problemas $^{15}$.

Duas abordagens foram adotadas na pesquisa: a) quantitativa - para diagnosticar a situação de saúde e a situação das Vigilâncias locais, por meio de dados dos sistemas de informação de interesse da saúde, dos dados fornecidos pela AGEVISA, das Secretarias Municipais de Saúde e dos Serviços de Vigilância Sanitária municipais, bem como pelo trabalho sobre a Situação dos Serviços de Vigilância Sanitária no país ${ }^{1}$; e b) qualitativa - visando identificar os problemas e fatores de risco por meio de entrevistas semiestruturadas com atores sociais, e por fotografias e visitas in loco para reconhecer o território, dimensionar os problemas sanitários e, assim, delinear o diagnóstico de campo dos municípios.

O estudo foi constituído por três momentos distintos, que, para efeito de sistematização, podem ser distinguidos por: a) momento 
anterior ao trabalho de campo, que incluiu a sistematização dos dados dos sistemas de informação de interesse da saúde, a elaboração dos instrumentos, a preparação teórica e prática dos pesquisadores e a organização da logística para o trabalho de campo; b) momento de observação e investigação do território, que orientou a abordagem qualitativa; e c) momento de sistematização e análise das informações geradas pela pesquisa.

O período de permanência da equipe nos municípios dependeu da complexidade do campo da Vigilância Sanitária local e da disponibilidade dos atores sociais para as entrevistas. O grupo formado por seis técnicos visitou serviços de saúde, indústrias de produção de açúcar e de mineração, áreas destinadas ao depósito de resíduos sólidos, abatedouros legalizados e clandestinos e estabelecimentos comerciais de alimentos. A equipe esteve presente nos dias de feira-livre e nos horários de maior movimento dos mercados públicos, permanecendo dois dias em cada município e quatro dias nos casos de João Pessoa e Campina Grande, os dois municípios do Estado com maior população e complexidade produtiva.

\section{Os instrumentos e seu emprego no desenvolvimento da pesquisa}

Para coleta e sistematização do material de cada um dos momentos da pesquisa foram elaborados quatro instrumentos:

- Diagnóstico preliminar. utilizado no momento anterior ao trabalho de campo. Nele foram registrados os dados disponíveis nos sistemas públicos de informação - características geo-econômicas, sociais, demográficas, os principais indicadores de saúde e o número de estabelecimentos sujeitos à Vigilância Sanitária no município.

Na pesquisa de campo, orientou a busca inicial dos principais problemas locais. Nas visitas aos municípios, estes dados foram apresentados primeiramente aos gestores municipais, que em boa parte os desconheciam e algumas vezes os refutaram por divergirem dos dados gerados por seus muni- cípios, apesar de estarem atualizados e disponibilizados em sistemas nacionais de informação.

- Diagnóstico Institucional dos Serviços de Vigilância Sanitária: utilizado no momento de observação e investigação do território, com o objetivo de sistematizar informações sobre estrutura e processos de trabalho da Vigilância municipal. Nele foram reunidas informações fornecidas pessoalmente pelos Secretários de Saúde e Coordenadores locais de Vigilância Sanitária, quando da visita da equipe ao município.

- Roteiro para entrevistas semiestruturadas com atores sociais: utilizado no momento de observação e investigação do território. Foi elaborado com a finalidade de provocar e estabelecer o diálogo entre entrevistador e entrevistado, e legitimar o processo posterior de planejamento.

As entrevistas semi-estruturadas constituem a base da metodologia da $\mathrm{ERP}^{12}$ e foram realizadas atendendo aos critérios de bom conhecimento local e representatividade nas áreas relacionadas à Vigilância Sanitária, com os seguintes atores sociais: a) institucionais - gestores e técnicos do Estado e dos municípios, outras autoridades municipais, representantes do Ministério Público e representantes do Conselho $\mathrm{Mu}$ nicipal de Saúde; b) representantes do setor regulado - indústria e comércio locais; c) formadores de opinião-lideranças políticas e representantes de empresas locais e regionais de comunicação social (jornalistas, radialistas); e d) consumidores de produtos e usuários de serviços.

A pesquisa totalizou 93 entrevistas, nas quais foram registradas informações basicamente qualitativas, visando subsídios para a análise de aspectos como: contexto político da Vigilância Sanitária, gestão, recursos financeiros, processos de trabalho e percepção dos fatores de risco existentes nos municípios. Das entrevistas foram destacadas a idéia central, os desdobramentos e as expressões-chave ${ }^{16}$, buscando-se identificar as prioridades contidas nos discursos dos entrevistados.

Na análise de cada município, os proble- 
mas e prioridades apontadas pela equipe da VISA e aqueles apontados por outros atores sociais foram separados por áreas de atuação: alimentos, meio ambiente, saúde do trabalhador, serviços de saúde, medicamentos, cosméticos, saneantes, criação de animais em perímetro urbano e hotéis, em busca de convergências e divergências de opiniões, sendo posteriormente reunidos na composição do diagnóstico final.

- Plano de leitura: utilizado no momento de sistematização e análise dos dados e informações geradas pela pesquisa. Foi elaborado com o objetivo de sintetizar os dados e as informações dos instrumentos anteriormente descritos sob duas dimensões: a) caracterização do município e b) análise da situação da Vigilância Sanitária local.

\section{Critérios para a análise dos resultados}

Após a leitura do material da pesquisa, as variáveis que compuseram os critérios de avaliação foram reunidas em duas categorias: forças restritivas e forças impulsoras para a efetividade dos Serviços de Vigilância Sanitária nos municípios analisados ${ }^{18}$.

Para efeito desta pesquisa, forças restritivas são consideradas as variáveis negativas referentes a determinadas condições apresentadas pela Vigilância Sanitária no que tange à estrutura, processos de trabalho, gestão, contexto político e recursos financeiros. As forças impulsoras encontram-se na positividade dessas variáveis, consideradas estruturantes para uma ação efetiva ${ }^{18}$.

$\mathrm{Na}$ análise de estrutura dos Serviços foram considerados os seguintes aspectos: edificações adequadas (incluindo facilidade de acesso e visibilidade); infraestrutura de suporte para fiscalização (material para coleta, acondicionamento e transporte de amostras para análise fiscal); equipamento de informática para uso exclusivo; veículo para uso exclusivo; equipamentos de comunicação (fax, telefone); fontes de consulta técnica (internet, livros, periódicos); recursos humanos suficientes; recursos humanos capacitados (para as funções que exercem); remuneração adequada (conforme percep- ção da equipe de VISA); plano municipal de capacitação; processos de capacitação realizados nos últimos três anos (para identificar o alcance das capacitações promovidas ou financiadas pela ANVISA, desde a sua criação); existência de lei que cria a Vigilância Sanitária no município; existência de termos administrativos (impressos) para aplicar a legislação sanitária; e existência de Código Sanitário Municipal.

No que tange a processos de trabalho, foram analisados: orientação jurídica para os processos administrativos sanitários (apoio do setor jurídico do município); uso de cadastro atualizado (cadastro mais recente feito há dois anos); uso de cadastro informatizado; informatização dos processos de trabalho (preenchimento de formulários, termos, documentos e comunicações internas); utilização do Laboratório Central de Saúde Pública - LACEN (envio de amostras em uma freqüência superior a uma vez por ano); conhecimento das atribuições legais da VISA municipal (competências e áreas de atuação); conhecimento da capacidade operacional diante das atribuições legais (dimensionamento dos recursos necessários para a execução das atribuições); clareza das atribuições por esfera de governo (competências e campos de atuação de cada esfera); utilização de dados epidemiológicos como orientadores da ação (indicadores epidemiológicos do município); conhecimento integral do papel da Vigilância Sanitária (fiscalização, informação, comunicação e educação sanitárias); e ações desenvolvidas predominantemente para as atividades além da concessão de licenças, alvarás e atendimento de denúncias (visando identificar a existência de planejamento, de monitoração e de outras ações pró-ativas).

Com relação à gestão, os aspectos analisados foram: apoio administrativo e operacional (pela Secretaria Municipal de Saúde); coordenação efetiva (responsável pelo Serviço presente e atuante); visibilidade das ações (analisada a partir das entrevistas realizadas com atores sociais); planejamento das ações (plano de trabalho); articulação com outros setores da Secretaria de Saúde 
(incluindo Setores de Zoonoses e Vetores, Assistência e Planejamento, excluindo Vigilância Epidemiológica); articulação intersetorial municipal (incluindo Secretarias de Obras, Limpeza Pública, Agricultura, Educação e Companhias de Água e Esgotos); articulação técnica com a VISA estadual (reuniões, consultas e orientações técnicas); articulação político-administrativa com a Vigilância Sanitária estadual (comunicação entre gestores); e articulação com a Vigilância Epidemiológica do município.

A análise do contexto político do Serviço de Vigilância Sanitária resultou das opiniões dos entrevistados pela pesquisa. Foram considerados: o conhecimento da importância das ações de Vigilância pelo gestor municipal (objetivos das ações de controle sanitário); atores sociais que conhecem a importância das ações de VISA (percepção dos objetivos das ações de controle sanitário); atores sociais que compreendem o papel de proteção e promoção da saúde da Vigilância (percepção da finalidade das ações de controle sanitário); atores sociais que entendem o exercício do poder de polícia pela VISA (reação dos atores sociais face à aplicação ou não de sanções); a missão da VISA que prevalece sobre a ação político-partidária (relacionada à interferência político-partidária local); a influência da VISA no contexto político-social (participação em arenas decisórias); e a integração da VISA com a comunidade (ações voltadas para a comunidade, participação em eventos locais).

No que tange aos recursos financeiros, foram analisados: os gestores que conhecem o valor do PAB/VISA e os compromissos decorrentes; os atores sociais que conhecem o valor do PAB/VISA e os compromissos decorrentes (destinado s especialmente a conselheiros municipais de saúde e vereadores); os recursos do PAB/VISA aplicados exclusivamente em ações de VISA; o conhecimento do Termo de Ajuste e Metas (termo firmado entre a ANVISA e o Estado que estabelece as metas de controle sanitário e o respectivo repasse de recursos); a transfe- rência de recursos financeiros pelo Estado; e os valores arrecadados em taxas e multas que retornam à VISA .

As informações foram transformadas em variáveis dicotômicas (excludentes: positivas ou negativas) de acordo com a avaliação dos pesquisadores que visitaram, observaram e coletaram dados em cada área. A análise e valoração de algumas variáveis dependeram da interpretação do observador, a partir de suas impressões, representações e informações coletadas. Outras variáveis, como, por exemplo, existência de veículo para uso exclusivo, tinham sua resposta positiva ou negativa segundo as condições objetivas que se apresentavam. Atribuindo a cada variável com resposta positiva o valor " 1 ", o valor máximo de cada critério resultou do somatório de todas as variáveis consideradas como positivas. Ou seja, atingindo $100 \%$ de seu valor máximo, quando todas as variáveis apresentavam resposta com valor "1".

A partir da modificação das variáveis nominais dicotômicas em variável numérica, aplicou-se a avaliação da adequabilidade de cada critério ${ }^{18}$, a partir do percentual de objetivos atingidos em relação ao esperado, ou a partir da adequação e do funcionamento de determinado Serviço em relação a um modelo considerado satisfatório ${ }^{17}$. Assim, considerou-se que um percentual superior ou igual a $80 \%$ de respostas positivas indicaria uma situação satisfatória; um percentual inferior a $80 \%$ e maior ou igual a $40 \%$ indicaria uma situação não satisfatória; e inferior a $40 \%$ de respostas positivas indicaria uma situação crítica para o Serviço de Vigilância Sanitária local.

\section{Resultados}

As variáveis elaboradas no plano de leitura foram consolidadas em cinco tabelas e analisadas por município pesquisado. Para efeito de sistematização, apresentamos a seguir os resultados agregados dos oito municípios: 
Análise da situação dos Serviços de Vigilância Sanitária dos municípios de João Pessoa, Campina Grande, Cabedelo, Bayeux, Santa Rita, Soledade, Juazeirinho e Patos, estado da Paraíba, julho de 2003:

[Status of Sanitary Surveillance Services of the municipalites of João Pessoa, Campina Grande, Cabedelo, Bayeux, Santa Rita, Soledade, Juazeirinho and Patos, State of Paraíba, July 2003:]

\section{Estrutura}

\begin{tabular}{lcc}
\hline Estrutura & Sim (\%) & Não (\%) \\
\hline Edificações adequadas & 50,0 & 50,0 \\
Infra-estrutura de suporte para fiscalização & - & 100,0 \\
Equipamento de informática para uso exclusivo & 37,5 & 62,5 \\
Veículo para uso exclusivo & 25,0 & 75,0 \\
Equipamento de comunicação & 37,5 & 62,5 \\
Fontes de consulta técnica & - & 100,0 \\
Recursos humanos suficientes & 25,0 & 75,0 \\
Recursos humanos capacitados & - & 100,0 \\
Remuneração adequada & - & 100,0 \\
Plano municipal de capacitação de RH & - & 100,0 \\
Processos de capacitação de RH realizados nos últimos três anos & - & 100,0 \\
Existência de lei que cria órgão de VISA & 100,0 & - \\
Existência de termos administrativos p/ aplicar a Legislação Sanitária & 75,0 & 25,0 \\
Existência de Código Sanitário Municipal & 87,5 & 12,5 \\
\hline
\end{tabular}

\section{Processos de trabalho}

\begin{tabular}{lcc}
\hline Processos de trabalho & Sim (\%) & Não (\%) \\
\hline Orientação jurídica para os processos administrativos sanitários & - & 100,0 \\
Uso de cadastro atualizado de estabelecimentos sujeitos a VISA & 37,5 & 62,5 \\
Uso de cadastro informatizado de estabelecimentos sujeitos a VISA & - & 100,0 \\
Informatização dos processos de trabalho & - & 100,0 \\
Utilização do Laboratório Central de Saúde Pública para análises & 12,5 & 87,5 \\
$\quad$ & 37,5 & 62,5 \\
laboratoriais & 12,5 & 87,5 \\
Conhecimento das atribuições legais da VISA Municipal & - & 100,0 \\
Conhecimento da capacidade operacional diante das atribuições legais & 100,0 \\
Clareza das atribuições por esfera de governo & 100,0 \\
Utilização de dados epidemiológicos como orientadores da ação de VISA & - & - \\
Conhecimento integral do papel da VISA & 25,0 & 75,0 \\
Ações de VISA desenvolvidas predominantemente para atividades & \\
além da concessão de licenças, alvarás e atendimento de denúncias & & \\
\hline
\end{tabular}

\section{Gestão}

\begin{tabular}{lcc}
\hline Gestão & Sim (\%) & Não (\%) \\
\hline Apoio administrativo e operacional & 25,0 & 75,0 \\
Coordenação efetiva & 25,0 & 75,0 \\
Visibilidade das ações de VISA & 25,0 & 75,0 \\
Planejamento das ações de VISA & 12,5 & 87,5 \\
Planejamento das ações de VISA vinculado à realidade sanitária & 12,5 & 87,5 \\
Articulação da VISAcom outros setores da Secretaria de Saúde & 12,5 & 87,5 \\
Articulação intersetorial municipal & 12,5 & 87,5 \\
Articulação técnica com a VISA Estadual & 12,5 & 87,5 \\
Articulação político-administrativa com a VISA Estadual & - & 100,0 \\
Articulação com a área de Vigilância Epidemiológica do município & - & 100,0 \\
\hline
\end{tabular}




\begin{tabular}{lcc}
\hline Contexto político & Sim (\%) & Não (\%) \\
\hline Prioridade para a VISA nas políticas municipais & - & 100,0 \\
Influência político-partidária potencializa as ações de VISA & 12,5 & 87,5 \\
Gestores Municipais conhecem a importância das ações de VISA & 37,5 & 62,5 \\
Atores sociais conhecem a importância das ações de VISA & 25,0 & 75,0 \\
Atores sociais compreendem o papel de proteção e promoção da VISA & 12,5 & 87,5 \\
Atores sociais entendem o poder de polícia da VISA & 25,0 & 75,0 \\
Missão da VISA prevalece sobre ação político-partidária & 12,5 & 87,5 \\
Influência da VISA no contexto político-social & - & 100,0 \\
Integração da VISA com a comunidade & - & 100,0 \\
\hline
\end{tabular}

\section{Recursos financeiros}

\begin{tabular}{lcc} 
Recursos financeiros & Sim (\%) & Não (\%) \\
\hline $\begin{array}{l}\text { Gestores conhecem o valor do PAB/VISA e compromissos } \\
\text { decorrentes }\end{array}$ & 75,0 & 25,0 \\
$\begin{array}{l}\text { Atores sociais conhecem o valor do PAB/VISA e compromissos } \\
\text { decorrentes }\end{array}$ & - & 100,0 \\
$\begin{array}{l}\text { Recursos PAB/VISA aplicados exclusivamente em ações de Visa } \\
\text { Conhecimento do conteúdo do Termo de Ajustes e Metas }\end{array}$ & 37,5 & 62,5 \\
$\begin{array}{l}\text { Transferência de recursos pelo Estado } \\
\text { Valores arrecadados em taxas e multas retornam à Visa }\end{array}$ & - & 100,0 \\
\hline
\end{tabular}

\section{Discussão}

As variáveis analisadas indicaram fatores restritivos importantes para o efetivo trabalho de controle sanitário nos municípios pesquisados, que destacamos a seguir.

No estudo, constatou-se a pouca articulação entre o trabalho da Vigilância Sanitária e o espaço sobre o qual ela atua. A observação parte do fato de que $100 \%$ das equipes desconheciam os dados socioeconômicos e epidemiológicos dos seus municípios e, por conseguinte, os fatores de risco por eles delineados.

O território de ação das Vigilâncias pesquisadas, independentemente do porte do município, circunscrevia-se ao recebimento de denúncias e cadastramento de estabelecimentos, em boa parte $(62,5 \%)$ desatualizado e em sua totalidade (100\%) não informatizado. Além disso, a pesquisa constatou que $100 \%$ dos Serviços de Vigilância Sanitária não possuíam infraestrutura de suporte para a fiscalização, em $100 \%$ destes os técnicos não tinham acesso a fontes de consulta técnica, não havia clareza das atri- buições por esfera de governo para $100 \%$ das equipes, $62,5 \%$ delas desconheciam as atribuições legais da própria VISA municipal, e 100\% demonstraram não ter conhecimento integral do papel da Vigilância Sanitária.

Embora 75\% dos Serviços dispusessem de Termos Administrativos, para notificação ou aplicação de sanções, raramente os utilizavam. As orientações e determinações eram predominantemente verbais e, no seu descumprimento, algumas VISAs evitavam os conflitos gerados pelas sanções. Surgiu em várias equipes a justificativa de que caberia à Vigilância Sanitária "moderna" o papel de educar, não o de punir. Comunicação e educação em saúde são fundamentais para as ações de Vigilância Sanitária ${ }^{6}$. Entretanto, ambas não excluem o dever de aplicar sanções adequadas e necessárias, porque a população ainda conta, principalmente, com a intervenção do Estado para a sua proteção, devido à realidade de boa parte dos municípios brasileiros - cujas relações sociais, econômicas e políticas envolvem práticas clientelistas e patrimonialistas, pouca parti- 
cipação social nos processos decisórios, baixa escolaridade, pouca informação, restrita capacidade de escolha e incipiente responsabilidade pública dos que produzem , comercializam e prestam serviços ${ }^{19,20}$.

Em decorrência da omissão do poderdever da Vigilância de preservar o interesse público por meio do controle sanitário, este acaba sendo efetuado pelo Ministério Público, que, conforme constatado nas entrevistas, tem se ocupado nos municípios com a criação de suínos em perímetro urbano, com restaurantes sem condições de higiene e com visitas e interdições de hospitais, demandas não resolvidas pela Vigilância Sanitária que acabam no Poder Judiciário ${ }^{2}$.

Como foi dito anteriormente, o território é também, e fundamentalmente, um espaço de poder ${ }^{3}$. Daí sua importância estratégica para as políticas públicas no enfrentamento de problemas e necessidades da população que o habita e o produz socialmente. Nessa perspectiva, constatou-se, entre os atores entrevistados, que $75 \%$ dos Serviços de Vigilância Sanitária não tinham visibilidade para a sociedade, $87,5 \%$ dos entrevistados desconheciam seu papel de proteção e promoção da saúde, $75 \%$ não entendiam o exercício de seu poder de polícia, para 87,5\% dos entrevistados a influência político-partidária prevalecia sobre a missão da Vigilância e, na totalidade (100\%) dos municípios pesquisados, a Vigilância Sanitária não tinha influência no contexto político-social.

Embora tenha sido comum a justificativa de recursos humanos insuficientes e pouco capacitados para a execução das ações de controle sanitário, foi possível verificar na pesquisa equipes com carga horária de 8 a 12 horas semanais de trabalho, acumulação de funções, capacitações concentradas em poucos técnicos e desarticuladas de um programa de aperfeiçoamento que priorize as necessidades locais e a integralidade das ações de Vigilância Sanitária.

Apesar de outros problemas de estrutura estarem presentes, os fatores restritivos relacionados à gestão também sobressaíram. Não foi identificado planejamento das ações de Vigilância Sanitária, e mesmo os 12,5\% dos serviços que elaboraram plano anual de trabalho, bem como os $12,5 \%$ dos que o fizeram vinculado à realidade sanitária, o realizaram mediante observações pessoais das equipes, sem o subsídio de dados epidemiológicos, sociais e econômicos do município, e sem o conhecimento sistematizado da cadeia de produção e consumo, bem como desarticulado dos atores e do contexto político e social do território.

Esta desarticulação da Vigilância Sanitária com o seu entorno, suscita Paulo Freire ${ }^{21}$ quando pergunta "A política que nós fazemos está a favor de quem?" Por desconhecer a realidade local e por não se reconhecer como ator institucional, social e político importante para a proteção e promoção do interesse público, foi possível constatar que a Vigilância Sanitária acaba por reforçar a exclusão social existente. Isto porque ela atua, em sua maioria, sobre o território do que é oficial ou legalizado. Por exemplo, na época da pesquisa, em um dos municípios visitados, somente $0,28 \%$ da população era abastecida por rede geral, e a atuação da Vigilância local no controle da água para consumo humano se dava somente sobre este percentual, ou seja, $99,72 \%$ da população não contava com o controle sanitário da água que consumia. Por que tamanha exclusão social no trabalho da Vigilância Sanitária?

Um outro exemplo foi a constatação da atuação incipiente sobre o comércio ambulante e abatedouros clandestinos, que em geral ofertavam alimentos a preços menores e tinham como consumidores a população com menor poder aquisitivo. O problema do comércio informal ou clandestino é complexo e requer articulação e políticas intersetoriais. Ao se depararem com ele, e com a população que dele se abastece, alguns Serviços de Vigilância Sanitária se omitem, e circunscrevem seu território de ação ao comércio legalizado, especialmente devido a pressões políticas e econômicas locais.

No estudo, o controle sanitário dos alimentos foi destacado como prioridade pelos atores sociais dos oito municípios, inclusive nos dois maiores municípios do Estado 
- João Pessoa e Campina Grande. As condições sanitárias dos mercados públicos, em muitos dos municípios visitados, reportavam às condições de higiene da Idade Média. Neles, além de alimentos em condições precárias de higiene e conservação, eram comercializados preparados (de uso tópico e oral) com alegação de indicação terapêutica. Por que preparados vendidos como medicamentos, por $\mathrm{R} \$ 1,00$, para a população de baixa renda, não demandam controle sanitário tal como os industrializados e comercializados em farmácias?

Estes mercados, mesmo nos pequenos municípios, reúnem um comércio variado, em parte de origem clandestina, em alguns municípios abastecem cerca de $40 \%$ da população ${ }^{2}$, em sua maioria de menor renda, e suas instalações dependem da atuação de vários setores municipais, desde a Vigilância Sanitária, as Secretarias de Agricultura, de Planejamento, de Obras e de Limpeza Públicas, até as Companhias de Água, Esgotos e Energia Elétrica. Ou seja, um trabalho de fiscalização e educação sanitárias que demanda conhecimento técnico, articulação intersetorial e influência política.

Paradoxalmente, mesmo com tal complexidade, o controle sanitário de alimentos é considerado uma atividade de baixa complexidade pela Vigilância Sanitária, conforme parâmetro originado da Portaria no 18/MS/ SAS de 21 de janeiro de 1999. Caberia perguntar o que são, para a Vigilância Sanitária, ações de baixa, média e alta complexidade? Qual o resultado desta classificação no incentivo ou falta de incentivo do controle sanitário de alimentos, especialmente nos municípios menores e economicamente mais pobres? Face à abrangência e especificidade das ações de Vigilância Sanitária, e à expertise já acumulada neste campo, ainda se justifica, conforme Lucchesi, "assimilar acriticamente os princípios e pressupostos pensados para a política de assistência, sem perceber que a natureza dos problemas e da intervenção necessária é substancialmente diversa, exigindo um debate próprio"? ?2

Um outro aspecto constatado diz respeito aos dados de mortalidade. No ano de
2003, a mortalidade proporcional por causas mal definidas, no Estado da Paraíba, era da ordem de $45,1 \%$ (27,5\% na Região Nordeste e $14,1 \%$ no Brasil $)^{23}$. Isto significa o desconhecimento de quase metade das mortes ocorridas naquele ano, na Paraíba, informação imprescindível para a definição de prioridades de intervenção. Ao pesquisar as causas deste dado, os fatores apontados na época tanto pelo Centro Nacional de Epidemiologia (Cenepi/MS) como pela Vigilância Epidemiológica do Estado foram o preenchimento incorreto das Declarações de Óbito (DO) pelos médicos e a ausência de um Sistema de Verificação de Óbitos no Estado. Deve-se considerar, ainda, tanto a possibilidade de insuficiente atendimento médico à população, como problemas na qualidade da assistência dos serviços de saúde.

Os dados referentes a intoxicações e envenenamentos por medicamentos, alimentos, saneantes, agrotóxicos e outros, diretamente ligados aos produtos sob controle sanitário, ainda são insuficientes. Além do problema da sub-notificação, as informações fornecidas pelo Sistema Nacional de Informações Tóxico-Farmacológicas (SINITOX/ FIOCRUZ), não identificam o município de ocorrência do agravo, mas os pólos regionais de atendimento (no caso da Paraíba, estão em Campina Grande e João Pessoa) ${ }^{24}$, o que dificulta a localização espacial das principais fontes de agravo e, conseqüentemente, o controle sanitário por parte da Vigilância Sanitária .

Sob o aspecto da informação como subsídio para o planejamento, o estudo constatou a dificuldade em reunir dados fidedignos tanto em relação à situação de saúde como ao campo de ação da Vigilância local. Além disso, a coleta e a sistematização dos dados epidemiológicos disponibilizados nos sistemas de informação não atendem plenamente às necessidades da Vigilância Sanitária, pois desde a coleta até a consolidação não prevêem o controle sanitário entre seus objetivos. Por outro lado, cabe lembrar também que os dados oficiais têm a tendência de excluir os segmentos marginalizados da população, reforçando a exclusão social ${ }^{12}$. 
Finalizando, apesar de se tratar de uma amostra de conveniência, ou seja, os municípios pesquisados não foram selecionados por aleatoriedade estatística ${ }^{4}$, pode-se sugerir que os resultados aqui apresentados são representativos da situação dos Serviços de Vigilância Sanitária do Estado da Paraíba. Porém, longe de destacar pontualmente problemas de Vigilância Sanitária de um Estado e se encerrar na exposição de aspectos a serem aprimorados, o trabalho teve como objetivo propor a intervenção crítica sobre o território por meio do planejamento participativo.

Nesse sentido, em decorrência das prioridades identificadas pela pesquisa, a AGEVISA realizou um conjunto de oficinas e seminários com municípios para a elaboração de uma agenda de compromissos para enfrentamento dos fatores restritivos encontrados, e para definição dos projetos a serem desenvolvidos. Por fim, um outro aspecto a ser ressaltado refere-se à disposição encontrada nas equipes dos municípios estudados para a melhoria do trabalho de suas Vigilâncias Sanitárias.

\section{Agradecimentos}

Os autores agradecem a decisiva contribuição de Luiz Carlos Pelizari Romero, Consultor Legislativo do Senado Federal, na concepção e análise da Pesquisa e do Projeto relatados neste artigo.

\section{Referências}

1. Lima LCW, Reis LGC. Levantamento da situação dos serviços de Vigilância Sanitária no país. Rio de Janeiro: mimeo, 2002.

2. Agência Nacional de Vigilância Sanitária. Projeto Redevisa - Planejamento Participativo em Vigilância Sanitária: Relato da experiência na Paraíba. Brasília; 2003.

3. Teixeira C. Planejamento Municipal em Saúde. Salvador: Ed. Instituto de Saúde Coletiva; 2001.

4. Pereira MG. Epidemiologia Teoria e Prática. Rio de Janeiro: Ed. Guanabara Koogan; 2003. p. 484, 342.

5. Costa EA. Vigilância Sanitária. Proteção e Defesa da Saúde. São Paulo: Ed. Hucitec e Sociedade Brasileira de Vigilância de Medicamentos; 1999.

6. Costa EA e Rozenfeld S. Constituição da Vigilância Sanitária no Brasil. In: Rozenfeld S. Fundamentos da Vigilância Sanitária. Rio de Janeiro: Ed. Fiocruz; 2000. p. 15-40.

7. Barcellos C. Organização do Espaço, Saúde e Qualidade de Vida. In: Seminário Nacional de Saúde e Ambiente no Processo de Desenvolvimento. Série Fiocruz: Eventos Científicos, 2. Rio de Janeiro: Ed. Fiocruz; 2000.

8. Lucchese G. Globalização e Regulação Sanitária: os Rumos da Vigilância Sanitária no Brasil [tese de doutorado]. Rio de Janeiro: Escola Nacional de Saúde Pública da Fiocruz; 2001.

9. Teixeira C e Paim J. Planejamento e programação de ações intersetoriais para a promoção da saúde e da qualidade de vida. Rev de Administração Pública 2000; 34: $63-80$.
10. Junqueira LAP. Intersetorialidade, transetorialidade e redes sociais na saúde. Revista de Administração Pública 2000; 34: 35-45.

11. Fundação Instituto Brasileiro de Geografia e Estatística. Censo 2000. http://www.ibge.gov.br (acessado em 05/Jan/2003).

12. Di Villarosa FN. A estimativa rápida e a divisão do território no Distrito Sanitário - Manual de Instruções Série desenvolvimento de serviços de saúde 11. Brasília: OPS Representação do Brasil; 1993.

13. Tancredi FB, Barrios SRL, Ferreira JHG. Planejamento em Saúde. São Paulo: Ed. Faculdade de Saúde Pública da USP; 1998.

14. Santos MRGS. A pesquisa de estimativa rápida: instrumento de relações públicas nas organizações.http://www.google.com.br (acessado em 23/04/2003).

15. Machado GB, Bunchaft A. Gestão territorial e planejamento sócio-ambiental participativo em regiões urbano-periféricas - o caso do município de feira de Santana Governo do Estado da Bahia (http:// www.google.com.br acessado em 23/04/2003.

16. Simioni AMC, Lefévre F, Pereira IMTB. Metodologia Qualitativa nas Pesquisas em Saúde Coletiva: Considerações Teóricas e Instrumentais. Série Monográfica no 2 São Paulo: Centro de Estudos e Pesquisas em Direito Sanitário, Departamento de Prática de Saúde Pública, Faculdade de Saúde Pública da USP; 1996. 
17. Pedrosa JI. Abordagem metodológica na análise de intervenções voltadas para mudanças nas organizações de saúde. Revista do Instituto Materno Infantil de Pernambuco 2000; 14: 38-46.

18. Moreno EM, Ojeda ENS, Anderson CF. Evaluación de servicios de salud materno-infantil: condiciones de eficiência em países de America Latina. In: Evaluación de servicios de salud materno infantil. Buenos Aires; 1989. (Oganización Panamericana de la Salud/ Organización Mundial de la Salud).

19. Silva AA. A Predação do Social. Niterói: Ed. Universidade Federal Fluminense; 1997.

20. Nunes E. A Gramática Política no Brasil. Clientelismo e insulamento burocrático. Rio de Janeiro:Ed. ENAP/ Zahar; 1997.
21. Freire P. Como trabalhar com o povo? Textos de Saúde Pública. São Paulo: Associação Paulista de Saúde Pública; 1983.

22. Lucchesi G. Vigilância Sanitária no Sistema Único de Saúde [ante-projeto de tese de doutorado]. Rio de Janeiro: Escola Nacional de Saúde Pública da Fiocruz. Mimeo; 1997.

23. Indicadores e Dados Básicos para a Saúde. Rede Interagencial de informações para a Saúde RIPSA; 2003.

24. Fundação Oswaldo Cruz. Estatística anual de casos de intoxicação e envenenamento. Sinitox. Rio de Janeiro; 2000 .

recebido em: 19/08/04

versão final reapresentada em: 12/11/04

aprovado em: 13/12/04 